\title{
Study of Dried and Calcinated Ceria Stabilized Zirconia Microspheres Morphology by Small- Angle X-Ray Scattering and Optical Microscopy
}

\author{
A. Patriati ${ }^{1 *}$, A. Insani ${ }^{1}$, N. Suparno ${ }^{1}$, E. Hutamaningtyas ${ }^{1}$, S. Soontaranoon ${ }^{2}$, \\ Mujamilah $^{1}$ \\ ${ }^{I}$ Center for Science and Technology of Advanced Materials, National Nuclear Energy Agency (BATAN), \\ Puspiptek Area, Serpong Tangerang, Banten 15314, Indonesia \\ ${ }^{2}$ Synchrotron Light Research Institute, 111 University Avenue, Muang District, Nakhon Ratchasima 30000, Thailand
}

\section{ARTICLE INFO}

Article history:

Received 31 December 2019

Received in revised form 25 January 2021

Accepted 27 January 2021

\section{Keywords:}

CSZ

SAXS

Microsphere

Nuclear fuel

External gelation

\begin{abstract}
A B S T R A C T
The knowledge of how to avoid crack at high temperature is crucial in fuel fabrication for Experimental Power Reactor or Reaktor Daya Eksperimental (RDE). This knowledge should be established and well-mastered by BATAN. RDE uses uranium dioxide as its nuclear fuel. However, uranium utilization for research purposes is heavily restricted. Therefore, the fabrication of ceria-stabilized zirconia (CSZ) microspheres as nuclear fuel surrogate was studied. In this work, the CSZ was prepared by external gelation with two different washing solutions, i.e. isopropyl alcohol (IPA) and propylene glycol methyl ether (PGME). The morphology in nano- and micro-scale of each CSZ microspheres from both variations was evaluated by small-angle $\mathrm{x}$-ray scattering (SAXS) and optical microscopy. The morphology of the CSZ microsphere after drying at $80{ }^{\circ} \mathrm{C}$ and calcination at $200{ }^{\circ} \mathrm{C}$ were observed to understand the structural change in those steps and to see the potential crack based on its morphology. There are two parameters that can prevent microspheres from cracking at high temperatures for the next process, i.e., porosity and gel texture. It was observed that IPA solution has a benefit as a washing solution as it can trigger more porosities in the microspheres, but less in gel texture.
\end{abstract}

(C) 2021 Atom Indonesia. All rights reserved

\section{INTRODUCTION}

Experimental Power Reactor or Reaktor Daya Eksperimental (RDE) is one of the National Nuclear Energy Agency of Indonesia's (BATAN) top priority programs from 2015 to 2019. Building a nuclear reactor will be a huge milestone in nuclear technology development in Indonesia. Therefore, the mastery of the technology of every part of the RDE is important, including its fuel element. RDE is a pebble bed-type high temperature gas-cooled reactor (HTGR) using TRISO (tristructural isotropic) as its fuel kernel [1]. The successful fabrication of TRISO fuel depends on the quality of each step. Kernel fabrication in TRISO is as important as the other steps.

\footnotetext{
*Corresponding author.

E-mail address: arum@batan.go.id

DOI: https://doi.org/10.17146/aij.2020.1023
}

Research and development on kernel fabrication have been done in BATAN. The kernel of RDE fuel is a microsphere of coated uranium dioxide $\left(\mathrm{UO}_{2}\right)$. The microsphere will be coated by a buffer layer, inner pyrolytic carbon, silicon carbide, and outer pyrolytic carbon. Before being coated, there are several conditions that need to be satisfied by the $\mathrm{UO}_{2}$ microsphere to be used in a fuel element. The microsphere must have excellent sphericity and does not crack at high temperature [2]. Cracking of the microsphere at high temperature has become an important issue as the kernel fabrication process must go through heating the microsphere at a temperature of more than $1000{ }^{\circ} \mathrm{C}$.

Since $\mathrm{UO}_{2}$ is a nuclear material, the research on $\mathrm{UO}_{2}$ kernel fabrication has many restrictions in terms of waste and radiation. Therefore, the research on kernel fabrication needs a surrogate material with similar properties as $\mathrm{UO}_{2}$. The surrogate material 
must possess similar crystal structure to $\mathrm{UO}_{2}$, i.e. fluorite with FCC crystal structure (Fm-3m). Metal oxide with fluorite structure is commonly found in oxide compounds of elements in actinide and lanthanide series. In this work, zirconium has been chosen as surrogate material because it is known as a good material for nuclear fuel surrogates [3-5]. Furthermore, cerium was added to stabilize the zirconium oxide, called ceria-stabilized zirconia (CSZ).

The CSZ microsphere can be synthesized by external or internal gelation. Many researchers reported that the external gelation method has many advantages. The CSZ microsphere that was synthesized by the aforementioned method obtained better sphericity, mono-dispersity, and higher crushing strength [6]. However, the quality of the CSZ microsphere is depended on the vibrating frequency during synthesis process [7].

Although the synthesis procedure of the CSZ microsphere has been widely reported, the technique to reproduce the perfect CSZ microsphere needs to be established. It was reported that there are several crucial parameters in CSZ synthesis by external gelation. Those parameters are vibrating frequency and $\mathrm{pH}$ conditions that will affect the size, uniformity, and smooth surface of the CSZ microsphere [7]. Therefore, the technique and knowledge in CSZ microsphere synthesis have to be well understood and mastered. The previous work on synthesizing the CSZ microsphere by the BATAN team using external gelation methods faced some problems [8]. One of the important factors that affect the endurance of the microsphere from cracking is the solution condition, including the washing solution. The solution used in the washing process will affect the morphology of the microsphere, which in turn influences the endurance of the CSZ microsphere from cracking on heating.

Therefore, in this works, the morphology of the CSZ microsphere in nano and micro scale was revealed. The morphology both after drying and after calcination will be evaluated to get more understanding of the effect of the washing solution on the quality of the CSZ microsphere. Propylene glycol methyl ether (PGME) and isopropyl alcohol (IPA), which are considered good for the external gelation method, were chosen as washing solutions.

Small-angle $\mathrm{x}$-ray scattering (SAXS) is a technique that utilizes the elastic scattering of an $\mathrm{X}$-ray beam from a sample. SAXS has the ability to explore the nano-structure of material based on the difference of their element's interaction with X-ray [9]. This study will be the first report on the nanostructure morphology of the CSZ microsphere. This information will give insight on revealing the potential cause of cracking in microsphere at high temperature. Meanwhile, optical microscopy can give information about material morphology in a micro-scale by magnification image. Thus, the nanoand micro-structure of the CSZ microsphere will be explored using SAXS and optical microscopy, respectively.

\section{METHODOLOGY}

\section{CSZ microsphere synthesis}

The materials used in this study were zirconium nitrate, cerium nitrate, polyvinyl alcohol (PVA), tetrahydro furfuryl alcohol (THFA), urea, ammonium hydroxide, isopropyl alcohol (IPA), and propylene glycol methyl ether (PGME). All chemicals were purchased from Merck and used without purification.

The external gelation method was chosen to synthesize the CSZ microsphere [10]. The synthesis was began by making broth. A mixture of zirconium nitrate and cerium nitrate was dissolved in deionized water, then a certain amount of urea was added under heating and vigorous stirring. Under this condition, urea was hydrolyzed and released free ammonia. The ammonia then reacted with $\mathrm{Zr}^{2+}$ and $\mathrm{Ce}^{3+}$, forming mixed metal oxide of $\mathrm{ZrO}_{2}$ and $\mathrm{Ce}_{2} \mathrm{O}_{3}$.

The PVA and THFA were then added to the mixture overnight. These polymers were added to make the gelation occurred in the solution. The gelation process was begun by transferring the broth into the reactor. In this reactor, the broth was dropped by vibration at a certain frequency to gain a perfect spherical droplet. The droplets were collected in ammonia solution. Finally, gel microspheres were separated from the ammonia solution by filtration.

The droplets were washed in deionized water. The next washing step was divided into two different solutions, IPA and PGME. In this work, the microspheres which were washed by IPA and PGME solution were called m-IPA and m-PGME, respectively.

The droplet as gel microspheres were then dried at $80{ }^{\circ} \mathrm{C}$ for both m-PGME and m-IPA. The dried microspheres were transferred to a crucible and then calcinated at $200{ }^{\circ} \mathrm{C}$.

\section{SAXS and OM characterization}

SAXS experiment was conducted at BL.3 in SLRI, Thailand. To achieve the desired $q$ range, the experiment was carried out at two detector positions, i.e. $4500 \mathrm{~mm}$ and $800 \mathrm{~mm}$. This configuration 
setting obtained q range from $0.07 \mathrm{~nm}^{-1}$ to $4 \mathrm{~nm}^{-1}$. The data reduction was done by using SAXSIT program [11] and then analyzed by Igor SANS Analysis program from NIST [12].

The microscopy measurement was acquired using an Olympus BX 51 optical microscope at BATAN, Indonesia. The experimental condition and procedure were identical with the previous work [13].

\section{RESULTS AND DISCUSSION}

The external gelation synthesis in this study utilized the hydrolysis of urea as the source of ammonia. The ammonia reacted with zirconium ion and cerium ion, became zirconium oxide and cerium oxide in the water solution system [2]. The organic compound, PVA and THFA, was added to make the broth of CSZ solution have certain viscosity to form a microsphere. The droplets were pre-hardened in the air with ammonia gas. This step is important to keep the sphericity of the microsphere in the following collision with the ammonia solution. Once broth droplets contacted with ammonia solution, they were solidified in a very short period.

The washing process with deionized water removed the remaining nitrate in the microsphere. The PGME or IPA was applied to wash or substitute the water in the microsphere. During drying process, PGME and IPA will evaporate faster than water. It results in a higher porosity in the microspheres. Higher porosity is vital to prevent cracking of the CSZ microspheres due to heating during calcination and sintering process. When the porous CSZ gel microspheres were heated, the gases from organics decomposition escaped without damaging the microspheres [14].

The analysis of the SAXS scattering profile of the m-IPA dried at $80{ }^{\circ} \mathrm{C}$ (Fig. 1a) obtained the existence of moieties with a diameter of around $3.5 \mathrm{~nm}$ and polydispersity of 0.42 . In this state, the CSZ microspheres consist of mixed metal oxide sol of $\mathrm{ZrO}_{2}$ and $\mathrm{Ce}_{2} \mathrm{O}_{3}$ (CSZ) which were covered by polymer as their matrix formed a porous microsphere. The small particles observed in the m-IPA system were the pores. The high polydispersity of those pores can happen as there were organic compounds surrounded the CSZ ceramics cluster. The organics prevented the CSZ ceramics growth and formed a porous system.

The size and polydispersity of the pores changed after calcination. It was observed from SAXS data (Fig. 1b) that after calcination of m-IPA at $200{ }^{\circ} \mathrm{C}$, the diameter of the pores was around $4 \mathrm{~nm}$ with a polydispersity of 0.38 . This data confirmed that some of the pores fused each other in the calcination process due to the incineration of organics in the microsphere [2]. The fusing of some neighboring pores increased the pore size after calcination.

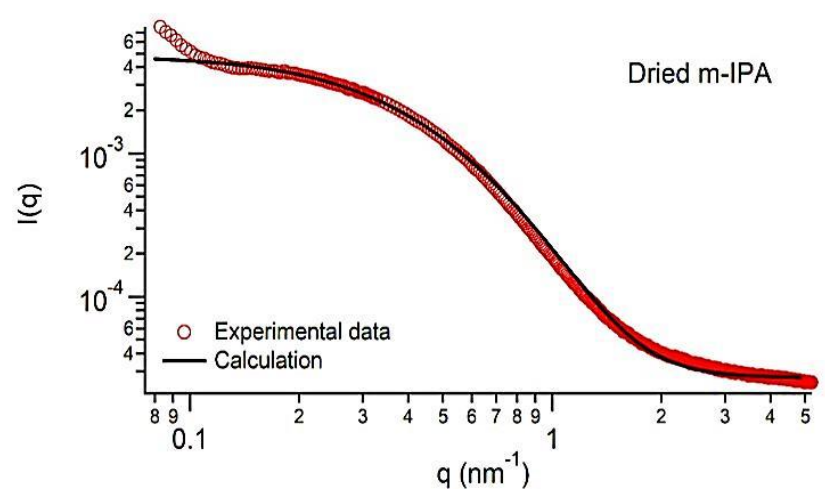

(a)

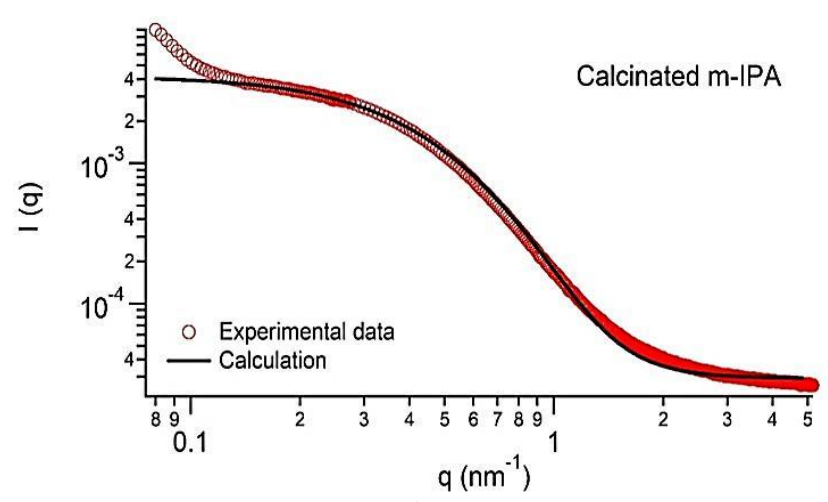

(b)

Fig. 1. SAXS scattering profile of CSZ microsphere with IPA as washing solution, (a) dried at $80^{\circ} \mathrm{C}$ and (b) calcinated at $200{ }^{\circ} \mathrm{C}$.

The same phenomenon was shown by analyzing the SAXS scattering profile (Fig. 2) of the m-PGME microsphere. The pores' size after the drying process was around $3.2 \mathrm{~nm}$ with a polydispersity of 0.42 . The size of the pores increased to around $3.7 \mathrm{~nm}$ and followed by the decrease in the polydispersity down to 0.38 . The pore size in the m-IPA microsphere was bigger than one in the m-PGME microsphere. The lower boiling point of IPA compared to PGME caused IPA molecules evaporated faster in the drying process. The faster evaporation created bigger pores in the microsphere. When IPA molecules in the microsphere were evaporated by heating, the organics and CSZ ceramics attempted to fill in the space. As IPA evaporated in a very short time, the organics and CSZ ceramics did not have sufficient time to fill it. Meanwhile, as PGME evaporated relatively slow compare to IPA, the pores formed after the drying process were relatively small. 
The upturn in the low q region indicated the existence of a bigger particle. In this case, the big particle was the CSZ ceramic itself. However, the size of the ceramic particle cannot be determined by SAXS, due to the limitation of the q range obtained from SAXS measurement. The SAXS scattering data could be analyzed to determine the size of a particle if there was a flat turn after the upturn.

Nevertheless, the SAXS scattering data was able to confirm that the size of CSZ ceramics particle in IPA washing solution was bigger than CSZ ceramics particle from PGME washing solution. It could be concluded from the turning point of the m-IPA microsphere scattering data. The turning point of the m-IPA microsphere occurred in the smaller $\mathrm{q}$ region compared to the turning point in the m-PGME microsphere. The turning point of scattered $x$-ray in the m-IPA and $\mathrm{m}$-PGME microsphere began at $\mathrm{q}$ around $0.15 \mathrm{~nm}^{-1}$ and $0.2 \mathrm{~nm}^{-1}$, respectively.

The IPA molecules were smaller compared to the PGME molecules. When the PGME molecules took place of water molecules in the microsphere gel particle, the bulkiness of the PGME molecules prevented the CSZ ceramics from growing. Consequently, the CSZ ceramics particles of m-PGME were smaller than one of m-IPA.

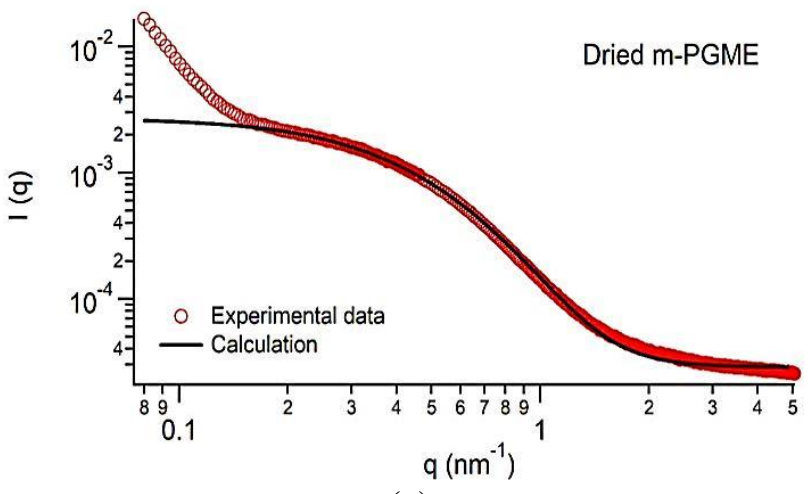

(a)

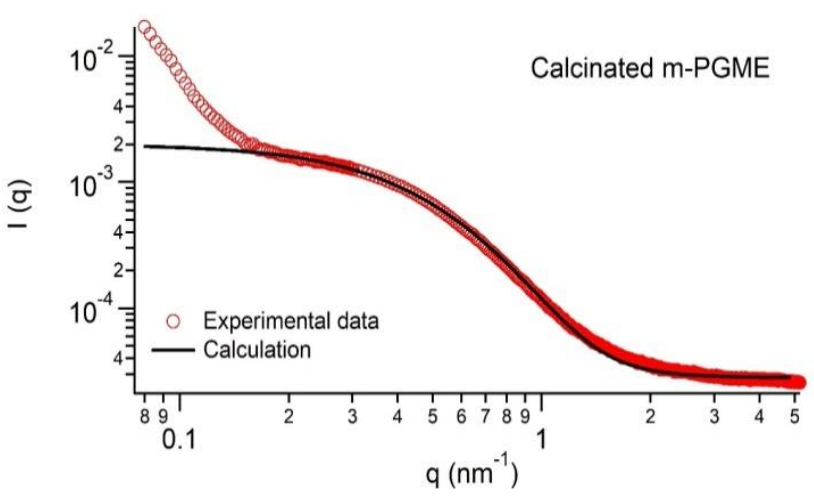

(b)

Fig. 2. SAXS scattering profile of CSZ microsphere with PGME as washing solution, (a) dried at $80{ }^{\circ} \mathrm{C}$ and (b) calcinated at $200{ }^{\circ} \mathrm{C}$
The increase of the homogeneity of the pore in the microsphere after the calcination process for both systems can be seen in the size distribution profile (Fig. 3). The narrowing of the distribution profile indicated that the range of size distribution of the pore was declining, which implies the increase of the pore size homogeneity. From this result, it can be assumed that some of the neighboring pores in the microsphere had fused each other. The fusing between pores seemed to happen for the smaller pore. Therefore, they formed a bigger pore and subsequently resulted in the decrease of the polydispersity after calcination.

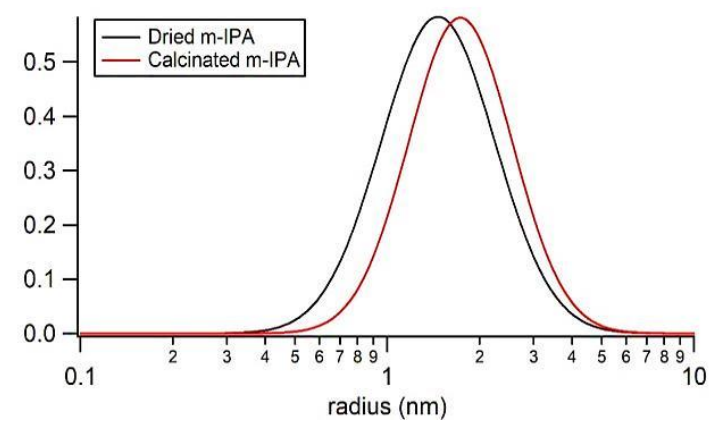

(a)

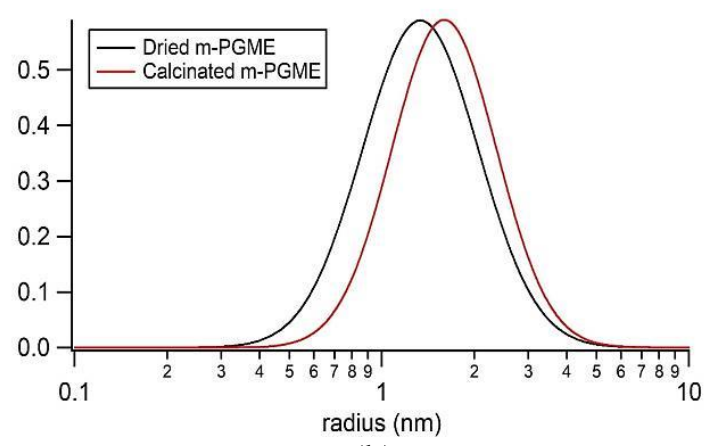

(b)

Fig. 3. The size distribution profile of CSZ microsphere with (a) IPA as washing solution, and

(b) PGME as washing solution.

Since the size of the pores inside CSZ microsphere with IPA as washing solution was smaller than PGME, while their polydispersity was similar, it gave a benefit for IPA as washing solution in this synthesis. Higher porosity gained with IPA as washing solution made the amorphous CSZ has good prevention on cracking [2].

The morphology of the m-IPA and m-PGME microsphere at a micrometer scale was studied with optical microscopy (OM). It is important to have a deep look at the microsphere at the micrometer scale. The sphericity and the gelation of the microsphere can be revealed by OM. The CSZ microsphere of both dried m-IPA and m-PGME had a similar sphericity value of around 1.02 , which is a good value, and it did not change after calcination. 
This result is better compared to the previous reports $[2,10]$.

The calcination process showed the rigid and firm microspheres which were synthesized by external gelation. The similar sphericity level of the CSZ microsphere for either m-IPA or m-PGME confirmed the independence of the CSZ microsphere sphericity with the type of the washing solution. The sphericity of the CSZ microsphere depended more on the synthesis parameters before washing. Since in this study those parameters were identical, the CSZ microsphere sphericity for both washing solution variations was similar.

The OM image (Fig. 4) provided information that the washing solution affected the perfection of the gelation of the CSZ microsphere. The line texture inside the CSZ microsphere was observed in both washing systems. However, the CSZ microsphere of m-IPA showed less apparent texture compared to the CSZ microsphere of m-PGME. The texture formed in CSZ microsphere due to incomplete gelation when the broth was dropped and rinsed in ammonia solution. When the CSZ microsphere beads were washed, the washing molecules took place the water molecule. The washing solution was removed from the CSZ microsphere by heating at low temperature (in this study $\left.\mathrm{t}=80{ }^{\circ} \mathrm{C}\right)$. Furthermore, the organics transformed from a gel into a solid-state. The gel which still existed in the CSZ microsphere after the drying process might come from some parts of the organics which stayed in gel form. The less apparent texture in m-IPA might happen due to the relatively small molecules of IPA. For its small size, the IPA molecules could insert into the organics' matrix more dispersedly. Consequently, the organics had more change to transform into solid as they have more surface in the drying process.

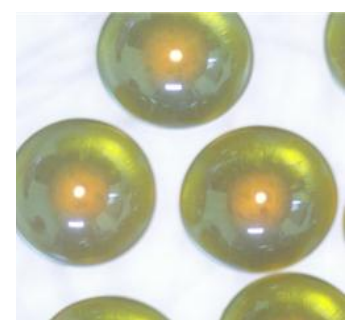

(a)

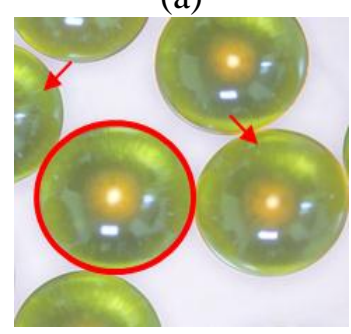

(c)

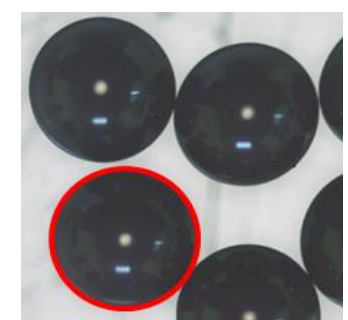

(b)

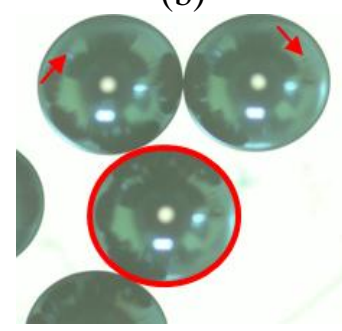

(d)
Fig 4. The OM image of CSZ microsphere of (a) dried m-IPA, (b) calcinated m-IPA, (c) dried m-PGME, and (d) calcinated mPGME as washing solution.
The experience with the previous work [8] showed that the gel texture inside the microsphere was the point of the crack. Increasing the gel texture in the microsphere means more potential cracking points. The gel texture inside the microsphere caused the CSZ microsphere to be fragile during sintering process. The remaining gel phase in the CSZ microsphere decreased the solidity of the microsphere itself. Therefore, IPA as a washing solution had a benefit on preventing the incomplete gelation inside the nanosphere.

\section{CONCLUSION}

Compared to the PGME, IPA as a washing solution had some advantages and earned better quality. The structure of the CSZ microsphere in nanoscale showed that IPA as a washing solution created bigger pores. It was beneficial for the microsphere as a fuel kernel as it would prevent the microsphere from cracking during sintering process. The study in microscale showed that IPA as a washing solution had an advantage in supporting the transformation of organics in the microsphere from a gel into a solid form. Incomplete gelation would create some gel texture inside the microsphere that could affect the microsphere. The microsphere would become fragile and cracked during sintering.

\section{ACKNOWLEDGMENT}

Authors acknowledge all staff in SLRI for helping and supporting in SAXS experiment. Authors also acknowledge Mr. Maryo for helping in the OM experiment. Thank you to the Ministry of Research and Technology and Higher Education of Indonesia for funding this research (INSINAS).

\section{AUTHOR CONTRIBUTION}

Arum Patriati and Andon Insani wrote the manuscript. Andon Insani, Nadi Suparno, and Mujamilah synthesized the sample. Evangelin Hutamaningtyas and Siriwat Soontaranoon did the SAXS experiment. Arum Patriati analyzed the SAXS data. Arum Patriati and Andon Insani did the OM measurement. Arum Patriati and Andon Insani equally contributed as the main contributors of this paper.

\section{REFERENCES}

1. P.H. Liem, T.M. Sembiring and H.N. Tran, Nucl. Eng. Des. 340 (2018) 245. 
2. W. Guogao, M. Jingtao, G. Yong et al., Int. J. Appl. Ceram. Technol. 13 (2016) 831.

3. S. Zhao, J. Ma, X. Zhao et al., Nucl. Eng. Des. 362 (2018) 110542.

4. R.D. Hunt, J.W. McMurray, G.W. Helmreich, et al., J. Nucl. Mater. (2020).

5. J. Aihara, S. Ueta, M. Honda et al., J. Nucl. Mater. 522 (2019) 32.

6. G. Wang, J. Ma, Y. Gao et al., J. Sol-Gel Sci. Technol. 78 (2016) 673.

7. G. Wang, J. Ma, Y. Gao et al., Key Eng. Mater. 697 (2016) 364.

8. R. Sukarsono, M. Rachmawati, S.R. Susilowati et al., J. Phys. Conf. Ser.
962 (2018) 1.

9. J. Li, A. Jiao, S. Chen et al., J. Mol. Struct. 1165 (2018) 391.

10. G. Wang, J. Ma, Y. Gao et al., J. Sol-Gel Sci. Technol. 78 (2016) 514.

11 S. Soontaranon and S. Rugmai, Chinese J. Phys. 50 (2012) 204.

12. S.R. Kline, J. Appl. Crystallogr. 39 (2006) 895.

13. A. Insani, A. Patriati, N. Suparno et al., J. Sains Mater. Indones. 20 (2019) 89. (in Indonesian)

14. R.D. Hunt, F.C. Montgomery and J.L. Collins, J. Nucl. Mater. 405 (2010) 160. 